\title{
Addictions and Personality Traits: Impulsivity and Related Constructs
}

\author{
Marci R. Mitchell • Marc N. Potenza
}

Published online: 4 January 2014

(C) Springer International Publishing AG 2014

\begin{abstract}
Behavioral tendencies that might be captured through self-report measures may provide insight into personality features that are associated with substance addictions. Recently, impulsivity and related constructs, such as sensationseeking, have been examined to help better understand their relationships with addictions. Here, we review recent findings that show links over developmental epochs between addictive behaviors and impulsivity, sensation-seeking, and other constructs that are theoretically linked. These findings have significant implications for generating improved treatments and interventions aimed at preventing the development of addictive disorders.
\end{abstract}

Keywords Impulsivity · Sensation-seeking · Substance use · Addiction $\cdot$ Personality Trait

\section{Introduction}

Personality features have long been associated with addictions. Recently, impulsive tendencies have been considered important in the psychopathologies of addictions. Impulsivity may contribute to a wide range of psychopathology, including bipolar disorder [1], attention deficit hyperactivity disorder [2],

M. R. Mitchell • M. N. Potenza $(\square)$

Department of Psychiatry, Yale University School of Medicine,

1 Church St, 7th floor, New Haven, CT 06510, USA

e-mail: marc.potenza@yale.edu

\section{N. Potenza}

Department of Neurobiology, Yale University School of Medicine, New Haven, CT, USA

M. N. Potenza

Child Study Center, Yale University School of Medicine,

New Haven, CT, USA borderline personality disorder [3], pathological behaviors associated with Parkinson's disease [4], and substance addictions [5]. This review will focus on recent studies (published within the last year) that investigate impulsivity and addictions.

Impulsivity has been described as rapid, unplanned behavior with little forethought of the consequences [6, 7]. Given this definition, some impulsivity may contribute to optimal decision-making and be advantageous in certain situations. However, high levels of impulsivity are generally maladaptive, resulting in adverse consequences, consistent with other definitions of impulsivity (e.g., "a predisposition toward rapid, unplanned reactions to internal or external stimuli with diminished regard to the negative consequences of these reactions to the impulsive individual or others") $[6,8]$. Impulsivity is a multidimensional construct that may be conceptually divided into at least three distinct forms: trait impulsivity, impulsive action, and impulsive choice [6,9]. Behavioral facets of impulsivity may be measured in multiple species, including rodents, nonhuman primates, and humans, using various tasks, many of which are directly translational (i.e., behavioral tasks exist for each species).

\section{Measures of Impulsivity and Related Constructs}

\section{Self-Report Assessments}

Impulsive personality traits (trait impulsivity) are generally assessed by self-report questionnaires, such as the Barratt Impulsiveness Scale (BIS-11) [10], which generates an overall measure of impulsivity and several subscale factors-for example, nonplanning, inattention, and motor (for review of impulsivity as a trait see Evenden [9]). Other questionnaires that contain measures of impulsivity or that are theoretically related to impulsivity, but are arguably less commonly used than the BIS-11, include the Schedule for Nonadaptive and 
Adaptive Personality (SNAP; [11]), Sensitivity to Punishment and Sensitivity to Reward Questionnaire, the Frontal Systems Behavior Scale, the Tridimensional Personality Scale [12], and the Eysenck Personality Inventory [13].

A separate yet arguably related construct is sensationseeking, which may be defined as "the need for varied, novel, and complex sensations and experiences..." [14] and is commonly measured by the Zuckerman Sensation-Seeking Scale [14]. Several of the self-report scales that are used to assess impulsivity also include questions to measure sensationseeking (and vice versa). For example, the ZuckermanKuhlman Personality Questionnaire [15] dedicates a portion of its questions to measuring impulsivity and another portion to sensation-seeking, and these constructs have at times been reported concurrently and at others separately.

Another commonly used self-report questionnaire that contains various subscales measuring both impulsivity and sensation-seeking is the Urgency, Premeditation, Perseverance, and Sensation-Seeking (UPPS) Impulsive Behavior Scale $[16,17]$. The original UPPS generated four subscales including Premeditation, Urgency, Perseverance, and Sensation-Seeking. Premeditation tendencies reflect individual differences in thinking through and reflecting on consequences before acting. More recently, in a revised version, urgency was separated into negative and positive urgencies. Urgency tendencies reflect individual differences in one's propensity to act impulsively in situations of high (negative or positive) affect. Perseverance tendencies reflect individual differences in remaining focused on tedious tasks. Sensationseeking tendencies reflect individual differences in excitement- or thrill-seeking. The extent to which these subscales represent various facets of impulsivity is debated as they may reflect separable personality traits that map in unique ways onto addictions $[16,17]$.

\section{Laboratory Assessments}

In the laboratory, impulsivity may be measured through impulsive action or impulsive choice. Impulsive action refers to a diminished ability to withhold a proponent response and is often referred to as "motor impulsivity" (for review see [2]). Impulsive action may be measured by the Stop-Signal Task, in which participants are required to make a rapid response to a cue and then, on certain trials and without warning, are also given a "stop" signal indicating that they should inhibit that response [18]. In some ways similar to the Stop-Signal Task is the Go/Nogo, in which participants are given two cues. The first is a cue to "go" and exert an action, and the second a "nogo" cue in which they must inhibit the action [19]. Individuals who have difficulty successfully inhibiting their response after the stop signal is presented are said to display greater impulsive action.
Impulsive choice is reflected in a preference for smaller, faster rewards over larger, delayed rewards. Impulsive choice may be measured in the laboratory with delay-discounting tasks, in which individuals are presented with choices between smaller, more immediate rewards and larger, but delayed rewards. Delay discounting describes the phenomenon during which the subjective value of rewards decreases with increasing delays before reward receipt, or as the reward or consequence of an action becomes farther into the future, the less influence the said reward or consequence has over an individual's ongoing behavior. Individuals who would be considered impulsive or showing high levels of impulsive choice generally choose the smaller, faster reward more often than the larger, delayed reward, especially when the choice of the small reward seems (objectively) disadvantageous (e.g., choice of $\$ 100$ today over $\$ 150$ tomorrow could be considered disadvantageous as a relatively short delay of gratification would result in substantially greater gains) [20,21].

The Stroop color-word interference test is a widely used cognitive-control task that is used to measure cognitive conflict and inhibition as individuals are presented with the name of a color (e.g., "blue") and are asked to name the color (not word name) when the word name is printed in either a congruent (e.g., blue) or incongruent (e.g., red) color [22]. The incongruent trials require the individual to inhibit one feature (e.g., reading the word), but still allow for the other feature (e.g., naming the colored ink). Reaction time on incongruent trials is typically slower than those for congruent trials and that is known as the "Stroop Effect" [22, 23].

\section{Self-Report Associations Between Impulsivity and Addiction}

\section{Diagnostic and Substance-Related Considerations}

Behavioral tendencies or personality traits, such as impulsivity and sensation-seeking, have been associated with various substance-use disorders relating to alcohol [24], tobacco [25], opiates [26], and cocaine [27]. For example, Ready et al. [28] found that disinhibition, impulsivity, and manipulative tendencies predicted both self- and other-reported substance-use problems. Disinhibition, impulsivity, and sensation-seeking are generally elevated in adolescence, but decrease as the life span progresses [29]. However, the nature of relationships between facets of impulsivity and substance use have been relatively difficult to disentangle in human studies as impulsivity may contribute to use and may be altered by use. Thus, more longitudinal studies are needed to determine if personality traits are associated with substance-use disorders prior to use and to what degree they are influenced by use. It is possible that drug use itself might result in long-lasting changes in impulsivity. A recent study by Hopwood et al. [30] 
investigated personality traits among individuals with no, current, or remitted substance-use-disorders using the Schedule for Nonadaptive and Adaptive Personality (SNAP; [11]). They found that patients with a substance-use disorder displayed greater negative temperament (particularly selfharm) and disinhibition (specifically impulsivity). Interestingly, only disinhibition differed between those who had never used, those who currently used, and those who had remitted; it was highest in those who currently used, followed by those in remission, followed by those who had never used [30]. Additionally, Meda et al. [32] showed that individuals who were at risk of developing a substance-use disorder or those who were already addicted displayed increased impulsivity on the BIS11 and during an experiential discounting task compared with control participants $[31,32]$. These data suggest that personality traits such as disinhibition are associated with substance use and the finding that even those in remission display greater disinhibition than those who have never used further emphasizes the importance of investigating whether disinhibition is a pre-existing factor in substance use and/or is induced or exaggerated by use. Additionally, traits may contribute to the way in which individuals respond to questions on self-report questionnaires, such that highly impulsive individuals may impulsively select an answer as opposed to spending time considering responses [33].

\section{Co-Occurring Disorders: Cocaine and Psychiatric Disorders}

It is not uncommon for individuals with substance-use disorders to have other comorbid mental disorders. It is often difficult to determine which aspects of an individual's personality can be attributed to each or are a result of the dual diagnosis. Zhornitsky et al. [34] investigated impulsivity (using the BIS-11) and sensation-seeking (using the Zuckerman Sensation-Seeking Scale) in individuals with substance-use disorders, schizophrenia, dual diagnoses, and healthy control subjects. They found that impulsivity was elevated in all three patient populations compared with control subjects and that sensation-seeking was elevated in those with substance-use disorders and dual diagnoses, but not in those with schizophrenia alone and thus appears independent of schizophrenia diagnosis (schizophrenia alone was related to social anhedonia) [34]. These data suggest that regardless of schizophrenia comorbidity, sensation-seeking is associated with substance-use disorders. In fact, sensation-seeking is commonly associated with substance use, especially interactions between sensation-seeking and impulsivity. For example, cocaine-dependent individuals score higher on trait impulsivity and sensation-seeking questionnaires [35]. They also display elevated trait impulsivity in both measures of cognitive and emotion-driven impulsivity [36•]. Cocaine-dependent individuals perform worse than healthy control subjects on neuropsychological measures of response inhibition (Stroop, go/no go) and in their ability to adjust to new contingencies (reversal); however, trait impulsivity was not related to response inhibition. Interestingly, duration of cocaine use was related to response perseveration (reversal) [37]; however, alcohol, as opposed to cocaine use, had a greater impact on inhibitory control [37]. Additionally, cocaine-dependent individuals score higher on both positive and negative urgency using the UPPS-P, and perform more poorly than nonaddicted comparison subjects on the 2-back working memory test and Stroop color-word interference task [36•]. In this study, the amount of cocaine consumed was negatively related to both working memory and response inhibition performance [38]. Taken together, these data suggest that cocaine dependence might be differentially related to facets of impulsivity and sensation-seeking; however, relationships between impulsivity and sensation-seeking are not limited to cocaine dependence.

\section{Alcohol}

Amongst undergraduate college drinkers, those who were considered hazardous drinkers by the Alcohol Use Disorders Identification Test (AUDIT) reported an earlier age at drinking onset and scored higher on the BIS-11, the Sensitivity to Reward scale of the Sensitivity to Punishment and Sensitivity to Reward Questionnaire, and the Disinhibition and Executive Dysfunction section of the Frontal Systems Behavior Scale [39]. Problem drinking in undergraduates is significantly related to negative urgency, sensation-seeking, and lack of premeditation [40]. Negative urgency, as measured by the UPPS$\mathrm{P}$, has been associated with deliberate self-harm, alcohol consumption, and disordered eating [41•] (of note, positive urgency has been related to alcohol consumption [42]). A metaanalysis investigating impulsivity and alcohol use, using the UPPS, found that impulsivity and alcohol use are related overall, and while all aspects of the UPPS are related to drinking frequency, there are differential relationships with other aspects of alcohol use. Lack of perseverance predicted drinking quantity, drinking problems were strongly related to both positive and negative urgency, and alcohol dependence was most associated with negative urgency and lack of planning [41•]. However, a study using the UPPS found that impulsivity (specifically lack of premeditation and sensationseeking subscales) was more related to illicit substance use than hazardous drinking in young adults (aged 18-25). Among the subscales, lack of premeditation and sensationseeking were the most closely related to illicit substance use and parental/peer use and psychological distress appeared to represent common risk factors for both hazardous drinking and illicit substance use [43]. Tomassini et al. [44] investigated Iowa Gambling Task (IGT) performance and BIS-11 scores in abstinent alcoholics and healthy control subjects. They found that abstinent individuals with alcoholism made 
significantly more disadvantageous choices, leading to poorer scores. IGT performance was negatively associated with BIS11 Non-Planning Impulsivity scores [44], suggesting that either poor IGT performance might lead to alcoholism or alcohol use can result in poor IGT performance lasting into abstinence. Hamilton et al. [45, 46] found that hazardous drinkers score higher on BIS-11 self-report measures of impulsivity than nondrinkers [45] and that greater levels of selfreported stress are associated with greater self-reported impulsivity (BIS-11) and drinking behavior [46], suggesting that better stress management might increase self-control.

\section{Attentional Bias, Impulsivity, and Substance Use Disorders}

Interestingly, relationships between impulsivity and/or sensation-seeking may be dependent on drug type. Marino et al. [47] investigated the relationship between trait impulsivity (as measured by the BIS-11) and sensation-seeking with analgesic opioid misuse risk using the Current Opioid Misuse Measure (COMM). They found that sensation-seeking was not associated with COMM scores, but that attentional impulsiveness (as measured by the BIS-11) was associated with misuse risk even after controlling for age [47]. This finding is particularly interesting as Coskunpinar and Cyders [48] recently conducted a meta-analytic review discussing the interactions among attentional bias, impulsivity, and substance use. The relationships between impulsivity and substance-related attentional bias do not appear to be moderated by substance type. The relationships also do not appear to be moderated by individual components or measures of trait impulsivity (e.g., nonplanning or motor impulsivity), or by different assessments of attentional bias used (i.e., the measurement scale used was not a factor). Importantly, the relationships between behavioral impulsivity and substance-related attentional biases were significantly stronger than the relationships between self-reported impulsivity and substance-related attentional biases [48]. While more studies are needed to determine the relationships between individual facets of impulsivity and sensation-seeking with various drugs of abuse, data indicate strong relationships between these personality traits and substance use. Furthermore, behavioral measures of impulsivity are also critical in understanding the underlying mechanisms and relationships between impulsivity and substance use. To begin to understand the underlying mechanisms, it is often important to implore translational approaches, as there are preclinical equivalents to behavioral measures of impulsivity in humans that can be utilized to further understand the relationships between impulsivity and substance use.

Translational Assessments of Impulsivity in Drug Addictions

Compared with non-abusing individuals, drug-abusing patients generally show greater impulsive choice on delay- discounting tasks (i.e., greater preference for smaller, faster rewards than larger, delayed rewards) and greater impulsive action on the stop-signal task (i.e., a diminished ability to inhibit a proponent response) [49-59]. Maladaptive decision-making associated with cocaine use can last well into abstinence. Heil et al. [53] investigated impulsive choice using a delay-discounting procedure and found no differences in impulsive choice between cocaine-abstinent and cocainedependent individuals, but that both groups were more impulsive than control participants. Heroin users display greater impulsive choice (i.e., greater discounting for delayed rewards), an effect that seemingly lasts after heroin use has ceased [60]; however, animal studies suggest that while heroin use can increase impulsive choice, it is not a long-lasting effect following abstinence [61, 62] (there is a persistent increase in impulsive action [63]). This apparent inconsistency may be explained by several factors. For example, heroinusing humans may be maintained on methadone; thus, while they are no longer using heroin, they are still chronically receiving opioids. In animal studies, animals undergo complete abstinence and are no longer exposed to any substance. Additionally, the consistency in which animals receive the drug, the number of times the animal goes into withdrawal, and/or the severity of withdrawal (i.e., animals are provided with the drug at around the same time every day, whereas drug-using humans generally have to seek and obtain the drug and can go days without administration) could represent factors contributing differentially to impulsivity across species. In addition to cocaine and heroin use, individuals with current or past alcoholism [24, 64, 65] and cigarette smoking (both adolescent and adult) show greater impulsivity on both selfreport and behavioral measures [66-71]. Taken together, these results suggest that drug-using (prior and current) individuals exhibit greater impulsive choice than non-using individuals and that this elevation in impulsive choice may exist long into abstinence; however, it is unclear if greater impulsive choice is a pre-existing disposition or a consequence of drug use, nor is it clear what mechanisms may underlie relationships between impulsive choice and drug use.

Animal studies have been utilized to investigate whether cocaine might cause the increased impulsivity observed with chronic use. There have been mixed findings as to whether experimenter-administered cocaine increases impulsivity [72-78]. For example, Paine et al. [75] administered cocaine to rats for 14 days and tested them in a stop-signal task and found no alterations in impulsive action. Moreover, Dalley et al. [63] tested rats in the five-choice serial-reaction-time task (5CSRTT) after exposure to cocaine and found no alterations in impulsive action. Therefore, there is little evidence to suggest that chronic cocaine administration in rodents increases impulsive action. However, there is evidence to suggest that experimenter-administered cocaine might lead to increases in impulsive choice in delay-discounting tasks 
[73-75, 77, 79]. For example, Simon et al. [79] tested impulsive choice following 3 weeks of abstinence from chronic cocaine administration and found that cocaine-exposed rats showed greater preference for smaller, more immediately available rewards, which suggests that cocaine exposure might generate long-lasting increases in impulsive choice. Similar findings exist when cocaine is self-administered as opposed to experimenter-administered [80]. However, Broos et al. [81] did not find that cocaine self-administration increased impulsive choice, perhaps because rats in this study consumed less cocaine than in other studies [80]. Consistent with this notion, data suggest that cocaine-induced increases in impulsive choice might depend on the amount of cocaine consumed, such that "high self-administering rats", which self-administer higher quantities of cocaine, show increased impulsive choice in a delay-discounting task following selfadministration, whereas "low-self-administering rats" do not (Mitchell et al., Intake-dependent 1027 effects of cocaine selfadministration on impulsive choice in a 1028 delay discounting task, Submitted).

While substantial data link impulsivity to substance use and addictions, including aspects like relapse vulnerability (for review see [33]), the role of impulsivity as an endophenotype or predisposing factor to drug use is arguably less clear. Some data suggest that abnormalities, dysregulation or differences in brain structure and function might account for both impulsivity and an increased propensity toward substance use and addictions.

\section{Impulsive Endophenotypes and Addiction: Developmental Considerations}

Although impulsivity is relevant across the lifespan, adolescence is a critical period in which impulsivity and sensationseeking are elevated [29] and drug use is often initiated [82]. Adolescents are more likely than adults or children to experiment with tobacco, alcohol, and illicit drugs, as well as to have unprotected sex and engage in reckless driving [29, $83-85]$. By 12 th grade, nearly $48 \%$ of America's youth have experimented with illicit drugs, nearly $60 \%$ have tried tobacco, and $71 \%$ have sampled alcohol [82]. However, substance use can be initiated at any point in life, and as demonstrated above, impulsivity is correlated with substance use in adult populations. In this section we will discuss impulsivity as related to adolescent and adult populations.

\section{Adolescence}

A recent longitudinal study by Castellanos-Ryan et al. [86 ${ }^{\bullet}$ ] investigated predictors (using the Eysenck ImpulsivenessVenturesomeness-Empathy scale) at age 12 and developmental tempo between ages 11 and 13 of future substance use between 'ages 15 and 16 in boys with low socioeconomic status. They found that both pubertal status (the development point at a particular age) at age 12 and the developmental tempo (the rate at which development occurs between ages 11 and 13) predicted increased levels of substance use in mid to late adolescence, suggesting that the faster the boys develop, the more likely they are to experiment with substances and have substance-use problems. Additionally, sensation-seeking partially mediated the relationship between pubertal status at age 12 and substance use (but not impulse control); however, developmental tempo was not related to either. Impulse control moderated the relationship between sensation-seeking and, in particular, marijuana use, such that higher sensationseeking scores were associated with greater marijuana use, but only in those boys who had lower impulse control. Similarly, another study also found that sensation-seeking was associated with increased alcohol- and cannabis-use problems [87, 88]. These data suggest that there are personality traits that may make individuals more vulnerable to substance use, and that these traits may exist prior to substance use and not result directly from use.

Mischel and colleagues have investigated mechanisms enabling delayed gratification or foregoing an immediate reward for a larger, but delayed one, and the implications of such behavior. In a famous experiment, sometimes referred to as "the marshmallow experiment", the ability for 4-year-old children to forego a single marshmallow while sitting in a room alone for $15 \mathrm{~min}$ for the reward of two marshmallows was tested $[89,90]$. This experiment has been replicated and follow-up studies on individuals tested at young ages suggest that preschool proficiency at delaying gratification might predict the capacity for cognitive control in adulthood. For example, the extent to which children delayed gratification significantly predicted subsequent scholastic aptitude test (SAT) scores and social and emotional cognition in adolescence [91, 92]. Researchers have continued to follow the individuals tested in the early experiments and their childhood delay-togratification performance has predicted education achievement, sense of self-worth, and ability to cope with stress in adulthood, all of which are factors associated with resilience against addictions [93]. Metcalfe and Mischel proposed "cool" and "hot" systems to explain the dynamics of resisting temptation where "hot" systems refer to emotionally charged processes that are under stimulus control and "cool" systems refer to rational, controlled processes [94, 95, 96•]. The tendency to delay gratification seems to be a relatively stable within-group trait over four decades, as individuals showing a greater propensity toward delayed gratification at age 4 also did so in mid-life [96•]. The group showing a greater propensity toward delay demonstrated greater recruitment of the inferior frontal gyrus (implicated in impulse control) in suppressing undesired actions and less ventral striatal activation when suppressing undesired "hot" responses involving happy 
face cues, suggesting greater cortical control over subcortical response in individuals tending to delay gratification [96 ${ }^{\circ}$. A study using intrinsic principle component analyses [97] during an inhibitory working-memory task suggested that the manner in which brain networks (particularly cortical networks) operate and interact might underlie long-lasting tendencies relating to delayed gratification [98]. Thus, these findings suggest that tendencies early in life relating to impulse control might hold long-term prognostic implications, and efforts aimed at enhancing self-control at early stages of development are important to consider in the prevention of addictions.

In another study, the Substance Use Risk Profile Scale (SURPS) was used to determine if certain personality profiles in adolescents aged 12-13 years were predictive of substance use a year later. Sensation-seeking and hopelessness were predictive of alcohol and tobacco use and sensation-seeking alone was predictive of marijuana use [99]. Using the same scale in another study, Castellanos-Ryan and colleagues [100] showed that impulsivity, sensation-seeking, and hopelessness were predictive of alcohol- and drug-use problems in young adolescents and adults, and that these factors were important correlates of other emotional and behavior problems in adolescence such as depression and conduct problems. Importantly, the SURPS subscales explained significant variance in all problem indicators after controlling for gender, age, and ethnicity, at baseline and 18 months later, even after controlling for baseline symptom measures. Impulsivity was the most consistent and strongest predictor of substance-use and conduct problems [100].

Sher and colleagues have investigated how impulsivity and alcohol and tobacco use in adolescence and young adulthood may change as individuals mature. They recorded baseline measures in impulsivity using questions from both the Tridimensional Personality Questionnaire [12] and the Eysenck Personality Inventory [13] and cigarette and alcohol use at age 18. Subjects were then reassessed at ages 25, 29, and 35 years. Individuals who stop smoking show the greatest decrease in impulsive behaviors between ages 25 and 35 [101•]. Additionally, individuals who show the most marked decreases in impulsivity also show the steepest decrease in alcohol consumption between ages 18 and 25 years [102, 103]. These data suggest that changes in self-reported impulsivity correlate with changes in substance use during young adulthood. As such, screening for impulsivity during this period may help identify high-risk individuals for addictions, and efforts to reduce impulsivity through psychotherapeutic or pharmacological mechanisms may help reduce young adult substance abuse.

Adolescents score higher than adults on a SensationSeeking Scale [104], and sensation-seeking has correlated with substance misuse, perhaps through an increased need for stimulation and high emotional states [105]. CastellanosRyan and Conrod [106] reported findings suggesting that choice impulsivity is only related to substance use when an individual also displays an externalizing disorder and that sensation-seeking and choice impulsivity are differentially associated with substance use. Martin et al. [107] found that sensation-seeking is increased in both males and females who report nicotine and alcohol use, and that sensation-seeking was also increased in males who reported marijuana use, with sensation-seeking mediating the relationship between pubertal development and drug use. Martin and colleagues [108] also found that sensation-seeking correlated with disruptive behaviors, such that individuals with disruptive behaviors display more sensation-seeking. In adults with and without ADHD, only scores on the BIS-11 attentional subscale distinguished the two groups [109], suggesting that the increased risk of substance use in adolescents with externalizing disorders, impulsivity might be a key feature underlying the relationship.

\section{Adulthood}

While impulsivity and substance use are strongly associated, it is often difficult to disentangle whether trait impulsivity is a cause or an effect of drug use in humans. Animal models have been useful in investigating temporal relationships and have suggested a bidirectional relationship such that impulsive animals show greater susceptibility to drug use and drug use may increase impulsivity (this is particularly true with stimulant use). Several clinical studies implicate impulsivity as a possible pre-disposing factor for substance use. Ersche et al. (2012) investigated brain structure in individuals with stimulant addiction, their unaffected biological siblings, and healthy unrelated control subjects [110]. They found that drug users and their biological siblings had similar impairments in the stop-signal reaction task and structural abnormalities that were distinctly different than those of unrelated healthy controls. These data suggest that the association between impulsivity and substance use might have a neurobiological familial trait in vulnerable individuals. Moreover, they also found that such impairments and abnormalities were more severe in the substance-using group, suggesting that aspects of impulsivity might represent an endophenotype. As a follow-up, Ersche et al. [111] investigated possible cognitive and personality endophenotypes for drug dependence. They found that the cognitive profiles of unaffected siblings were characterized by deficits in executive function such as working memory and mental planning. Similar profiles of impaired response regulation have been reported in adults with a family history of alcohol dependence [112] and in children with drug-dependent parents $[113,114]$. Ersche et al. [111] also suggested that their data might indicate reduced emotional functioning in the sibling pairs, as reflected by high levels of trait anxiety and stress sensitivity. Consistent with this notion, the concept of negative urgency has been proposed that describes a personality trait of impulsive actions in response to intense negative affect [17]. It may be possible that cognitive deficits, elevations in impulsivity, and different/abnormal brain structure might be shared in family 
members prone to addictions, and these differences may predate exposure to stimulant drugs and predispose to the development of stimulant dependence.

Recreational cocaine users with and without familial histories of addiction may exhibit distinctive brain and behavioral phenotypes [115•]. Both recreational users and stimulantdependent individuals report high levels of sensationseeking and display altered gray matter volumes in the orbitofrontal cortex compared with unrelated healthy controls. Both reduced volume in the orbitofrontal cortex and dysfunction in decision-making in adolescence have been associated with the onset of substance use [116, 117], possibly increasing the risk of developing dependence [118]. A recent animal study investigated risk-taking in adolescent rodents as a predictor of future cocaine self-administration in adulthood and found a bidirectional relationship between risk-taking and cocaine self-administration such that greater risk-taking was predictive of greater cocaine intake and cocaine intake in turn resulted in elevations in risk-taking [119]. Risk-taking has been linked to ventral striatal structure and function in adolescent youth, with substance-abusing youth showing relatively diminished ventral striatal activation and greater risk-taking [120]. Taken together, data suggest that tendencies relating to impulsivity might be linked to brain neurobiology, and that these relationships exist early in life and might be altered by drug exposure.

\section{Translational Investigations of Endophenotypes}

There is evidence for trait-like impulsive phenotypes, relating to impulsive action [121] and impulsive choice [81, 122], which occur naturally in rodents and have been useful for investigating impulsivity as a predisposing factor for drug use. Studies in rodents indicate that such 'trait' impulsivity might predict addiction-related behaviors, although this relationship is dependent on impulsivity sub-type and drug class [119, 121-124]. Rats selected for impulsive action show greater self-administration of various substances such as cocaine [121], nicotine [125], alcohol [126], and methylphenidate [127], though not heroin [128]. Trait impulsive choice may predict both the acquisition and escalation of cocaine selfadministration [122, 129] (notably, choice impulsivity may predict resistance to extinction and enhanced relapse propensity to both nicotine [125] and cocaine [81]). A recent study investigated early and prolonged exposure to delayed rewards by separating adolescent rats into fixed-delay, progressivedelay, and no-delay exposure groups and then assessed future alcohol self-administration [130]. Early and prolonged exposure (either fixed- or progressive-delayed groups, compared with no exposure to delay) resulted in less impulsive choice. Rats that were in the fixed-delay group self-administered more alcohol than either the progressive-delay or the no-delay group [130].

\section{Impulsivity and Treatment Outcome}

Impulsivity and Inhibitory Control as Predictors of Treatment Outcome

Measures of impulsivity and inhibition are not only predictive of or associated with substance use, they may also predict treatment outcome. For example, delay discounting has predicted treatment abstinence in treatment-seeking adolescent marijuana smokers [131] and adult cigarette smokers [132]. Additionally, other behavioral measures of impulsivity and related constructs (e.g., discounting tasks and the Stroop) in treatment-seeking adult smokers and cocaine-dependent individuals have been predictive of treatment abstinence [23, 133]. While most studies using impulsivity as a predictor of abstinence are conducted in treatment-seeking participants, a study by Dallery and Raiff [134] investigated delay discounting as a predictor of cigarette smoking in a laboratory model of abstinence reinforcement in a group of smokers that was not treatment-seeking. After a 3-h deprivation period, participants were asked to complete a delay-discounting task and could earn progressively increasing amounts of money for each 30-s period of abstinence from smoking in each of three sessions (control, $\$ 5.00$ for low sessions, and $\$ 20.00$ for high sessions). Results showed that both low and high conditions increased the tendency to smoke relative to the control session. During the low condition, higher rates of delay discounting predicted cigarette smoking [134], such that individuals who decided to smoke had higher rates of delay discounting than those who decided to abstain. These data suggest that inhibitory control is an important component to the ability to remain abstinent and that improving an individual's inhibitory control may provide more promising treatment outcome.

Impulsivity and Inhibitory Control as Tools to Improve Abstinence

Recently, there has been a focus on investigating whether practicing or improving inhibitory behavior may be beneficial in improving treatment abstinence and preventing relapse. Muraven [135] split smokers who were planning on quitting into two groups. The first practiced two small acts of selfcontrol for 2 weeks prior to quitting and the second practiced a task that increased their self-awareness of self-control without actually exercising self-control for 2 weeks prior to quitting. They found that individuals who practiced self-control remained abstinent longer than those who practiced selfawareness but not self-control, and that practicing selfcontrol increased the odds of quitting by a factor of 1.56 [135]. In addition to smoking cessation, inhibitory training has also been used to reduce alcohol abuse. Houben and colleagues have conducted several studies investigating 
whether improving working memory and inhibitory control can reduce alcohol intake. They had problem drinkers perform a working memory training task (or control task) over the course of 25 days. They found that training working memory improved working memory and reduced alcohol intake for more than 1 month after cessation of training. Interestingly, automatic impulse level was a significant moderator of the indirect effect of working memory training on alcohol use [136]. Additionally, Houben and colleagues have developed a beer go/no-go paradigm during which participants are assigned one of two conditions. The first is a beer/no-go condition in which participants perform a go/no-go task that consistently pairs no-go trials with alcohol-related stimuli to increase response inhibition for alcohol-related stimuli. The second condition is a beer/go condition in which participants were required to respond to the alcohol-related stimuli during the go/no-go task. They found that heavy drinkers assigned to the beer/no-go condition showed a significant reduction in both implicit attitudes toward alcohol and weekly alcohol intake the week following training compared with before go/ no-go training [137, 138]. Jones and Field [139] modeled a stop-signal task after the beer go/no-go task (from Houben et al.) in which participants were required to press one button for alcohol-related stimuli and another button for neutral stimuli on go trials and then refrain from pressing either button on stop-signal trials. Results suggest that training reduced alcohol consumption in the laboratory, but did not reduce self-reported drinking the week following training [139]. Differences in findings between the two studies may be related to differences in stimuli presented (Jones et al. presented both alcoholrelated and neutral stimuli) and/or the nature of the task (go/ no-go vs stop-signal). Regardless of differences, taken together, the data suggest that tasks that improve inhibitory control might provide promise in increasing and maintaining abstinence. Curiously, Garavan, Foxe, and colleagues [140, 141] investigated inhibitory control in abstinent cocaine-dependent individuals (average abstinence was 32.3 weeks) and observed a potential "recovery" of the inhibitory systems such that while current cocaine users show deficits in inhibitory control, abstinent cocaine-dependent participants do not show differences while performing inhibitory control tasks compared with control participants. These data provide additional evidence supporting the role of inhibitory control over drug use in treatment outcome and suggest that the relationships between these factors should be investigated further.

\section{Conclusions}

Data indicate that impulsivity and sensation-seeking are related to substance use (regardless of type of substance). However, the degree to which different aspects of impulsivity or related constructs specifically predispose to or are the result of use of specific substances remains to be determined more precisely. While specific traits may increase propensity toward substance use, additional longitudinal studies are needed to determine how potential fluctuations in facets of impulsivity and sensation-seeking might correlate with substance-use behaviors and disorders. Considering how aspects of impulsivity might change during treatment (and might represent a potential target for treatment) also requires additional investigation. Additional research should also investigate heritability factors relating to impulsivity. As noted previously [48], behavioral measures of impulsivity will also be critical for advancing our knowledge and understanding of the relationships between impulsivity and substance use. Findings from such studies should help advance the development of improved and targeted prevention, treatment, and policy efforts.

Acknowledgments Supported by: R01DA020908 (MNP), P20DA027844 (MNP), Connecticut Department of Mental Health and Addiction Services, the Connecticut Mental Health Center and the National Center for Responsible Gaming (MNP). M.R. Mitchell is supported by T32DA007238 (Petrakis, PI). The funding agencies did not have input into the content of the manuscript.

\section{Compliance with Ethics Guidelines}

Conflict of Interest Marci Mitchell has no conflicts. Marc Potenza has received financial support or compensation for the following: consulting and advising for Boehringer Ingelheim, Lundbeck and Ironwood; having financial interests in Somaxon; research support from the National Institutes of Health, Veteran's Administration, Mohegan Sun Casino, the National Center for Responsible Gaming and its affiliated Institute for Research on Gambling Disorders, and Forest Laboratories pharmaceuticals; participating in surveys, mailings or telephone consultations related to drug addiction, impulse control disorders or other health topics; consulting for gambling and legal entities on issues related to addictions or impulse control disorders; providing clinical care in the Connecticut Department of Mental Health and Addiction Services Problem Gambling Services Program; performing grant reviews for the National Institutes of Health and other agencies; guest-editing journal sections; giving academic lectures in grand rounds, CME events, and other clinical or scientific venues; and generating books or book chapters for publishers of mental health texts.

Human and Animal Rights and Informed Consent This article does not contain any studies with human or animal subjects performed by the author.

\section{References}

Papers of particular interest, published recently, have been highlighted as:

- Of Importance

1. Swann AC. Impulsivity in mania. Curr Psychiatry Rep. 2009;11(6): 481-7.

2. Winstanley CA, Eagle DM, Robbins TW. Behavioral models of impulsivity in relation to ADHD: translation between clinical and preclinical studies. Clin Psychol Rev. 2006;26(4):379-95. 
3. Bornovalova MA, Lejuez CW, Daughters SB, et al. Impulsivity as a common process across borderline personality and substance use disorders. Clin Psychol Rev. 2005;25(6):790-812.

4. Housden CR, O'Sullivan SS, Joyce EM, et al. Intact reward learning but elevated delay discounting in Parkinson's disease patients with impulsive-compulsive spectrum behaviors. Neuropsychopharmacology. 2010;35(11):2155-64.

5. Ersche KD, Turton AJ, Pradhan S, et al. Drug addiction endophenotypes: impulsive versus sensation-seeking personality traits. Biol Psychiatry. 2010;68(8):770-3.

6. Moeller FG, Barratt ES, Dougherty DM, et al. Psychiatric aspects of impulsivity. Am J Psychiatry. 2001;158(11):1783-93.

7. Winstanley CA. The utility of rat models of impulsivity in developing pharmacotherapies for impulse control disorders. Br J Pharmacol. 2011;164(4):1301-21.

8. Potenza MN, de Wit H. Control yourself: alcohol and impulsivity. Alcohol Clin Exp Res. 2010;34(8):1303-5.

9. Evenden J. Impulsivity: a discussion of clinical and experimental findings. J Psychopharmacol. 1999;13(2):180-92.

10. Patton JH, Stanford MS, Barratt ES. Factor structure of the Barratt impulsiveness scale. J Clin Psychol. 1995;51(6):768-74.

11. Clark LA. Manual for the Schedule of Nonadaptive and Adaptive Personality, Edited. Minneapolis, MN: University of Minnesota Press; 1993.

12. Sher KJ, Wood MD, Crews TM, Vandiver PA. The Tridimensional Personality Questionnaire-Reliability and Validity Studies and Derivation of a Short-Form. Psychol Assess. 1995;7(2):195-208.

13. Eysenck HJ, Eysenck SBG. Manual of the eysench personality questionnaire, Edited. Educational and Industrial Testing Services: San Diego; 1968

14. Zuckerman M, Neeb M. Sensation seeking and psychopathology. Psychiatry Res. 1979;1(3):255-64.

15. Zuckerman M, Kuhlman DM, Joireman J, et al. A comparison of three structural models for personality: the big three, the big five, and the alternate five. J Pers Soc Psychol. 1993;65:757-68.

16. Whiteside SP, Lynam DR, Miller JD, Reynolds SK. Validation of the UPPS impulsive behaviour scale: a four-factor model of impulsivity. Eur J Personal. 2005;19(7):559-74.

17. Whiteside SP, Lynam DR. The Five Factor Model and impulsivity: using a structural model of personality to understand impulsivity. Personal Individ Differ. 2001;30:669-89.

18. Greenberg LM, Waldman ID. Developmental normative data on the test of variables of attention (T.O.V.A.). J Child Psychol Psychiatry. 1993;34(6):1019-30.

19. Hogg J, Evans PLC. Stimulus Generalization Following ExtraDimensional Training in Educationally Subnormal (Severely) Children. Br J Psychol. 1975;66(May):211-24.

20. Adriani W, Laviola G. Elevated levels of impulsivity and reduced place conditioning with d-amphetamine: two behavioral features of adolescence in mice. Behav Neurosci. 2003;117(4):695-703.

21. Steinberg L, Graham S, O'Brien L, et al. Age differences in future orientation and delay discounting. Child Dev. 2009;80(1):28-44.

22. MacLeod CM. Half a century of research on the Stroop effect: an integrative review. Psychol Bull. 1991;109(2):163-203.

23. Mitchell MR, Balodis IM, DeVito EE, et al. A preliminary investigation of Stroop-related intrinisic connectivity in cocaine dependence: associations with treatment outcomes. Am J Drug Alcohol Abuse. 2013;39(6):392-402.

24. Petry NM. Delay discounting of money and alcohol in actively using alcoholics, currently abstinent alcoholics, and controls. Psychopharmacology (Berl). 2001;154(3):243-50.

25. Kassel JD, Shiffman S, Gnys M, et al. Psychosocial and personality differences in chippers and regular smokers. Addict Behav. 1994;19(5):565-75.

26. Madden GJ, Petry NM, Badger GJ, Bickel WK. Impulsive and self-control choices in opioid-dependent patients and non-drug- using control participants: drug and monetary rewards. Exp Clin Psychopharmacol. 1997;5(3):256-62.

27. Garavan H, Hester R. The role of cognitive control in cocaine dependence. Neuropsychol Rev. 2007;17(3):337-45.

28. Ready RE, Watson D, Clark LA. Psychiatric patient- and informant-reported personality: predicting concurrent and future behavior. Assessment. 2002;9(4):361-72.

29. Spear LP. The adolescent brain and age-related behavioral manifestations. Neurosci Biobehav Rev. 2000;24(4):417-63.

30. Hopwood CJ, Morey LC, Skodol AE, et al. Pathological personality traits among patients with absent, current, and remitted substance use disorders. Addict Behav. 2011;36(11):1087-90.

31. Reynolds B, Schiffbauer R. Measuring state changes in human delay discounting: an experiential discounting task. Behav Processes. 2004;67(3):343-56.

32. Meda SA, Stevens MC, Potenza MN, et al. Investigating the behavioral and self-report constructs of impulsivity domains using principal component analysis. Behav Pharmacol. 2009;20(5-6):390-9.

33. Verdejo-Garcia A, Lawrence AJ, Clark L. Impulsivity as a vulnerability marker for substance-use disorders: review of findings from high-risk research, problem gamblers and genetic association studies. Neurosci Biobehav Rev. 2008;32(4):777-810.

34. Zhornitsky S, Rizkallah E, Pampoulova T, et al. Sensationseeking, social anhedonia, and impulsivity in substance use disorder patients with and without schizophrenia and in non-abusing schizophrenia patients. Psychiatry Res. 2012;200(2-3):237-41.

35. Vonmoos M, Hulka LM, Preller KH, et al. Differences in selfreported and behavioral measures of impulsivity in recreational and dependent cocaine users. Drug Alcohol Depend. 2013;133(1): 61-70.

36. Torres A, Catena A, Megias A, et al. Emotional and non-emotional pathways to impulsive behavior and addiction. Front Hum Neurosci. 2013;7:43. The authors investigated self-reported and behavioral impulsivity in humans with cocaine dependence or pathological gambling and those with neither condition to determine relationships with substance and nonsubstance addictions.

37. Fernandez-Serrano MJ, Perales JC, Moreno-Lopez L, et al. Neuropsychological profiling of impulsivity and compulsivity in cocaine dependent individuals. Psychopharmacology (Berl). 2012;219(2):673-83.

38. Albein-Urios N, Martinez-Gonzalez JM, Lozano O, et al. Comparison of impulsivity and working memory in cocaine addiction and pathological gambling: Implications for cocaine-induced neurotoxicity. Drug Alcohol Depend. 2012;126(1-2):1-6.

39. Lyvers M, Duff H, Basch V, Edwards MS. Rash impulsiveness and reward sensitivity in relation to risky drinking by university students: potential roles of frontal systems. Addict Behav. 2012;37(8):940-6.

40. Adams ZW, Kaiser AJ, Lynam DR, et al. Drinking motives as mediators of the impulsivity-substance use relation: pathways for negative urgency, lack of premeditation, and sensation seeking. Addict Behav. 2012;37(7):848-55.

41. Coskunpinar A, Dir AL, Cyders MA. Multidimensionality in Impulsivity and Alcohol Use: A Meta-Analysis Using the UPPS Model of Impulsivity. Alcohol Clin Exp Res. 2013;37(9):144150. This article emphasizes the importance of investigating impulsivity as a multifaceted construct and investigating attentionalbiases in substance dependence.

42. Dir AL, Karyadi K, Cyders MA. The uniqueness of negative urgency as a common risk factor for self-harm behaviors, alcohol consumption, and eating problems. Addict Behav. 2013;38(5):2158-62.

43. Shin SH, Chung Y, Jeon SM. Impulsivity and substance use in young adulthood. Am J Addict. 2013;22(1):39-45.

44. Tomassini A, Struglia F, Spaziani D, et al. Decision making, impulsivity, and personality traits in alcohol-dependent subjects. Am J Addict. 2012;21(3):263-7. 
45. Hamilton KR, Sinha R, Potenza MN. Hazardous drinking and dimensions of impulsivity, behavioral approach, and inhibition in adult men and women. Alcohol Clin Exp Res. 2012;36(6):958-66.

46. Hamilton KR, Ansell EB, Reynolds B, et al. Self-reported impulsivity, but not behavioral choice or response impulsivity, partially mediates the effect of stress on drinking behavior. Stress. 2013;16(1):3-15.

47. Marino EN, Rosen KD, Gutierrez A, et al. Impulsivity but not sensation seeking is associated with opioid analgesic misuse risk in patients with chronic pain. Addict Behav. 2013;38(5):2154-7.

48. Coskunpinar A, Cyders MA. Impulsivity and substance-related attentional bias: A meta-analytic review. Drug Alcohol Depend. 2013;133(1):1-14.

49. Bickel WK, Marsch LA. Toward a behavioral economic understanding of drug dependence: delay discounting processes. Addiction. 2001;96(1):73-86.

50. Coffey SF, Gudleski GD, Saladin ME, Brady KT. Impulsivity and rapid discounting of delayed hypothetical rewards in cocainedependent individuals. Exp Clin Psychopharmacol. 2003;11(1): $18-25$.

51. Colzato LS, van den Wildenberg WP, Hommel B. Impaired inhibitory control in recreational cocaine users. PLoS One. 2007;2(11): e1143.

52. Fillmore MT, Rush CR. Impaired inhibitory control of behavior in chronic cocaine users. Drug Alcohol Depend. 2002;66(3):265-73.

53. Heil SH, Johnson MW, Higgins ST, Bickel WK. Delay discounting in currently using and currently abstinent cocainedependent outpatients and non-drug-using matched controls. Addict Behav. 2006;31(7):1290-4.

54. Johnson MW. An Efficient Operant Choice Procedure for Assessing Delay Discounting in Humans: Initial Validation in Cocaine-Dependent and Control Individuals. Exp Clin Psychopharmacol. 2012;20(3):191-204.

55. Li CS, Milivojevic V, Kemp K, et al. Performance monitoring and stop signal inhibition in abstinent patients with cocaine dependence. Drug Alcohol Depend. 2006;85(3):205-12.

56. Moreno-Lopez L, Catena A, Fernandez-Serrano MJ, et al. Trait impulsivity and prefrontal gray matter reductions in cocaine dependent individuals. Drug Alcohol Depend. 2012;25(3):208-14.

57. Kirby KN, Petry NM, Bickel WK. Heroin addicts have higher discount rates for delayed rewards than non-drug-using controls. J Exp Psychol Gen. 1999;128(1):78-87.

58. Kirby KN, Petry NM. Heroin and cocaine abusers have higher discount rates for delayed rewards than alcoholics or non-drugusing controls. Addiction. 2004;99(4):461-71.

59. Lejuez CW, Bornovalova MA, Daughters SB, Curtin JJ. Differences in impulsivity and sexual risk behavior among inner-city crack/cocaine users and heroin users. Drug Alcohol Depend. 2005;77(2):169-75.

60. Nielsen DA, Ho A, Bahl A, et al. Former heroin addicts with or without a history of cocaine dependence are more impulsive than controls. Drug Alcohol Depend. 2012;124(1-2):113-20.

61. Schippers MC, Binnekade R, Schoffelmeer AN, et al. Unidirectional relationship between heroin self-administration and impulsive decision-making in rats. Psychopharmacology (Berl). 2012;219(2):443-52.

62. Harty SC, Whaley JE, Halperin JM, Ranaldi R. Impulsive choice, as measured in a delay discounting paradigm, remains stable after chronic heroin administration. Pharmacol Biochem Behav. 2011;98(3):337-40.

63. Dalley JW, Laane K, Pena Y, et al. Attentional and motivational deficits in rats withdrawn from intravenous selfadministration of cocaine or heroin. Psychopharmacology (Berl). 2005;182(4):579-87.

64. Lejuez CW, Magidson JF, Mitchell SH, et al. Behavioral and biological indicators of impulsivity in the development of alcohol use, problems, and disorders. Alcohol Clin Exp Res. 2010;34(8): $1334-45$.

65. Vuchinich RE, Simpson CA. Hyperbolic temporal discounting in social drinkers and problem drinkers. Exp Clin Psychopharmacol. 1998;6(3):292-305.

66. Reynolds B, Patak M, Shroff P, et al. Laboratory and self-report assessments of impulsive behavior in adolescent daily smokers and nonsmokers. Exp Clin Psychopharmacol. 2007;15(3):264-71.

67. Odum AL, Baumann AA. Cigarette smokers show steeper discounting of both food and cigarettes than money. Drug Alcohol Depend. 2007;91(2-3):293-6.

68. Kobiella, A., S. Ripke, N.B. Kroemer, et al. Acute and chronic nicotine effects on behaviour and brain activation during intertemporal decision making. Addict Biol. 2013. doi:10.1111/ adb. 12057.

69. Reynolds B, Richards JB, Horn K, Karraker K. Delay discounting and probability discounting as related to cigarette smoking status in adults. Behav Processes. 2004;65(1):35-42.

70. Mitchell SH. Measures of impulsivity in cigarette smokers and non-smokers. Psychopharmacology (Berl). 1999;146(4):455-64.

71. Ohmura Y, Takahashi T, Kitamura N. Discounting delayed and probabilistic monetary gains and losses by smokers of cigarettes. Psychopharmacology (Berl). 2005;182(4):508-15.

72. Duva SM, Silverstein SM, Spiga R. Impulsivity and risk-taking in co-occurring psychotic disorders and substance abuse. Psychiatry Res. 2011;186(2-3):351-5.

73. Dandy KL, Gatch MB. The effects of chronic cocaine exposure on impulsivity in rats. Behav Pharmacol. 2009;20(5-6):400-5.

74. Logue AW, Tobin H, Chelonis JJ, et al. Cocaine decreases selfcontrol in rats: a preliminary report. Psychopharmacology (Berl). 1992;109(1-2):245-7.

75. Paine TA, Dringenberg HC, Olmstead MC. Effects of chronic cocaine on impulsivity: relation to cortical serotonin mechanisms. Behav Brain Res. 2003;147(1-2):135-47.

76. Winstanley CA. The orbitofrontal cortex, impulsivity, and addiction: probing orbitofrontal dysfunction at the neural, neurochemical, and molecular level. Ann N Y Acad Sci. 2007;1121:639-55.

77. Zuo Y, Wang X, Cui C, et al. Cocaine-induced impulsive choices are accompanied by impaired delay-dependent anticipatory activity in basolateral amygdala. J Cogn Neurosci. 2012;24(1):196-211.

78. Bornovalova MA, Daughters SB, Hernandez GD, et al. Differences in impulsivity and risk-taking propensity between primary users of crack cocaine and primary users of heroin in a residential substance-use program. Exp Clin Psychopharmacol. 2005;13(4):311-8.

79. Simon NW, Mendez IA, Setlow B. Cocaine exposure causes longterm increases in impulsive choice. Behav Neurosci. 2007;121(3): 543-9.

80. Mendez IA, Simon NW, Hart N, et al. Self-administered cocaine causes long-lasting increases in impulsive choice in a delay discounting task. Behav Neurosci. 2010;124(4):470-7.

81. Broos N, Diergaarde L, Schoffelmeer AN, et al. Trait impulsive choice predicts resistance to extinction and propensity to relapse to cocaine seeking: a bidirectional investigation. Neuropsychopharmacology. 2012;37(6):1377-86.

82. NIDA. InfoFacts: High School and Youth Trends. 2010; Available from: http://www.nida.nih.gov/infofacts/hsyouthtrends.html.

83. Chambers RA, Taylor JR, Potenza MN. Developmental neurocircuitry of motivation in adolescence: a critical period of addiction vulnerability. Am J Psychiatry. 2003;160(6):1041-52.

84. Arnett JJ, Offer D, Fine MA. Reckless driving in adolescence: 'state' and 'trait' factors. Accid Anal Prev. 1997;29(1):57-63.

85. Arnett JJ. Adolescent storm and stress, reconsidered. Am Psychol. 1999;54(5):317-26.

86. Castellanos-Ryan N, Parent S, Vitaro F, et al. Pubertal development, personality, and substance use: A 10-year longitudinal study 
from childhood to adolescence. J Abnorm Psychol. 2013;122(3): 782-96. Such longitudinal studies are important in understanding how periods in development such as adolescence might contribute to substance use and abuse later in life.

87. Kaynak O, Meyers K, Caldeira KM, et al. Relationships among parental monitoring and sensation seeking on the development of substance use disorder among college students. Addict Behav. 2013;38(1):1457-63.

88. Doran N, Sanders PE, Bekman NM, et al. Mediating influences of negative affect and risk perception on the relationship between sensation seeking and adolescent cigarette smoking. Nicotine Tob Res. 2011;13(6):457-65.

89. Mischel W, Ebbesen EB, Zeiss AR. Cognitive and attentional mechanisms in delay of gratification. J Pers Soc Psychol. 1972;21(2):204-18.

90. Mischel W, Shoda Y, Rodriguez MI. Delay of gratification in children. Science. 1989;244(4907):933-8.

91. Mischel W, Shoda Y, Peake PK. The nature of adolescent competencies predicted by preschool delay of gratification. J Pers Soc Psychol. 1988;54(4):687-96.

92. Shoda Y, Mischel W, Peake PK. Predicting adolescent cognitive and self-regulatory competences from preschool delay of gratification: Identifying dianostic conditions. Dev Psychol. 1990;26(6): 978-86.

93. Ayduk O, Mendoza-Denton R, Mischel W, et al. Regulating the interpersonal self: strategic self-regulation for coping with rejection sensitivity. J Pers Soc Psychol. 2000;79(5):776-92.

94. Metcalfe J, Mischel W. A hot/cool-system analysis of delay of gratification: dynamics of willpower. Psychol Rev. 1999;106(1): 3-19.

95. Abelson RP. Computer simulation of "hot cognition". In: Tomkins SS, Mesick S, editors. Computer Simulation of Personality. New York: Wiley; 1963. p. 277-302.

96. Casey BJ, Somerville LH, Gotlib IH, et al. Behavioral and neural correlates of delay of gratification 40 years later. Proc Natl Acad Sci U S A. 2011;108(36):14998-5003. This follow-up study investigates how self-control around age 4 years relates to brain function in middle adulthood.

97. Yourganov G, Chen X, Lukic AS. et al.:Dimensionality estimation for optimal detection of functional networks in BOLD fMRI data. Neuroimage. 2011;56(2):531-43.

98. Berman MG, Yourganov G, Askren MK, et al. Dimensionality of brain networks linked to life-long individual differences in selfcontrol. Nat Commun. 2013;4:1373

99. Malmberg M, Kleinjan M, Vermulst AA, et al. Do substance use risk personality dimensions predict the onset of substance use in early adolescence? A variable- and person-centered approach. J Youth Adolesc. 2012;41(11):1512-25.

100. Castellanos-Ryan N, O'Leary-Barrett M, Sully L, Conrod P. Sensitivity and specificity of a brief personality screening instrument in predicting future substance use, emotional, and behavioral problems: 18-month predictive validity of the Substance Use Risk Profile Scale. Alcohol Clin Exp Res. 2013;37 Suppl 1:E281-90.

101. Littlefield AK, Sher KJ. Smoking desistance and personality change in emerging and young adulthood. Nicotine Tob Res. 2012;14(3):338-42. This study was an exention of references 103 and 104, which place particular importance on following impulsivity throughout the lifespan to determine how such fluctuations might relate to addictive behaviors.

102. Littlefield AK, Sher KJ, Steinley D. Developmental trajectories of impulsivity and their association with alcohol use and related outcomes during emerging and young adulthood I. Alcohol Clin Exp Res. 2010;34(8):1409-16.

103. Littlefield AK, Sher KJ, Wood PK. Is "maturing out" of problematic alcohol involvement related to personality change? J Abnorm Psychol. 2009;118(2):360-74.
104. Zuckerman M, Eysenck S, Eysenck HJ. Sensation seeking in England and America: cross-cultural, age, and sex comparisons. J Consult Clin Psychol. 1978;46(1):139-49.

105. Comeau N, Stewart SH, Loba P. The relations of trait anxiety, anxiety sensitivity, and sensation seeking to adolescents' motivations for alcohol, cigarette, and marijuana use. Addict Behav. 2001;26(6):803-25.

106. Castellanos-Ryan N, Conrod PJ. Personality correlates of the common and unique variance across conduct disorder and substance misuse symptoms in adolescence. J Abnorm Child Psychol. 2011;39(4):563-76.

107. Martin CA, Kelly TH, Rayens MK, et al. Sensation seeking, puberty, and nicotine, alcohol, and marijuana use in adolescence. J Am Acad Child Adolesc Psychiatry. 2002;41(12):1495-502.

108. Martin CA, Kelly TH, Rayens MK, et al. Sensation seeking and symptoms of disruptive disorder: association with nicotine, alcohol, and marijuana use in early and mid-adolescence. Psychol Rep. 2004;94(3 Pt 1):1075-82.

109. Crunelle CL, Veltman DJ, van Emmerik-van K. Oortmerssen, et al.: Impulsivity in adult ADHD patients with and without cocaine dependence. Drug Alcohol Depend. 2013;129(1-2):18-24.

110. Ersche KD, Jones PS, Williams GB, et al. Abnormal brain structure implicated in stimulant drug addiction. Science. 2012;335(6068): $601-4$.

111. Ersche KD, Turton AJ, Chamberlain SR, et al. Cognitive dysfunction and anxious-impulsive personality traits are endophenotypes for drug dependence. Am J Psychiatry. 2012;169(9):926-36.

112. Acheson A, Richard DM, Mathias CW, Dougherty DM. Adults with a family history of alcohol related problems are more impulsive on measures of response initiation and response inhibition. Drug Alcohol Depend. 2011;117(2-3):198-203.

113. Dawes MA, Tarter RE, Kirisci L. Behavioral self-regulation: correlates and 2 year follow-ups for boys at risk for substance abuse. Drug Alcohol Depend. 1997;45(3):165-76.

114. Tarter RE, Kirisci L, Mezzich A, et al. Neurobehavioral disinhibition in childhood predicts early age at onset of substance use disorder. Am J Psychiatry. 2003;160(6):1078-85.

115. Ersche KD, Jones PS, Williams GB, et al. Distinctive personality traits and neural correlates associated with stimulant drug use versus familial risk of stimulant dependence. Biol Psychiatry. 2013;74(2):137-44. As an expansion of Ersches Science article (see ref 111), this study examined sensation-seeking and impulsive and compulsive personality features as related to stimulant addictions.

116. Cheetham A, Allen NB, Whittle S, et al. Orbitofrontal volumes in early adolescence predict initiation of cannabis use: a 4-year longitudinal and prospective study. Biol Psychiatry. 2012;71(8): 684-92.

117. Xiao L, Bechara A, Grenard LJ, et al. Affective decision-making predictive of Chinese adolescent drinking behaviors. J Int Neuropsychol Soc. 2009;15(4):547-57.

118. Bechara A. Risky business: emotion, decision-making, and addiction. J Gambl Stud. 2003;19(1):23-51.

119. Mitchell, M.R., V.G. Weiss, B.S. Beas, et al.: Adolescent risk taking, cocaine self-administration, and striatal dopamine signaling. Neuropharmacology. doi:10.1038/npp.2013.295.

120. Schneider S, Peters J, Bromberg U, et al. Risk taking and the adolescent reward system: a potential common link to substance abuse. Am J Psychiatry. 2012;169(1):39-46.

121. Dalley JW, Fryer TD, Brichard L, et al. Nucleus accumbens D2/3 receptors predict trait impulsivity and cocaine reinforcement. Science. 2007;315(5816):1267-70.

122. Perry JL, Larson EB, German JP, et al. Impulsivity (delay discounting) as a predictor of acquisition of IV cocaine selfadministration in female rats. Psychopharmacology (Berl). 2005;178(2-3):193-201. 
123. Belin D, Mar AC, Dalley JW, et al. High impulsivity predicts the switch to compulsive cocaine-taking. Science. 2008;320(5881): 1352-5.

124. Poulos CX, Le AD, Parker JL. Impulsivity predicts individual susceptibility to high levels of alcohol self-administration. Behav Pharmacol. 1995;6(8):810-4.

125. Diergaarde L, Pattij T, Poortvliet I, et al. Impulsive choice and impulsive action predict vulnerability to distinct stages of nicotine seeking in rats. Biol Psychiatry. 2008;63(3):301-8.

126. Radwanska K, Kaczmarek L. Characterization of an alcohol addiction-prone phenotype in mice. Addict Biol. 2012;17(3): 601-12.

127. Marusich JA, Bardo MT. Differences in impulsivity on a delaydiscounting task predict self-administration of a low unit dose of methylphenidate in rats. Behav Pharmacol. 2009;20(5-6):447-54.

128. McNamara R, Dalley JW, Robbins TW, et al. Trait-like impulsivity does not predict escalation of heroin self-administration in the rat. Psychopharmacology (Berl). 2010;212(4):453-64.

129. Anker JJ, Perry JL, Gliddon LA, Carroll ME. Impulsivity predicts the escalation of cocaine self-administration in rats. Pharmacol Biochem Behav. 2009;93(3):343-8.

130. Stein JS, Johnson PS, Renda CR, et al. Early and prolonged exposure to reward delay: effects on impulsive choice and alcohol self-administration in male rats. Exp Clin Psychopharmacol. 2013;21(2):172-80.

131. Stanger C, Ryan SR, Fu H, et al. Delay discounting predicts adolescent substance abuse treatment outcome. Exp Clin Psychopharmacol. 2012;20(3):205-12.

132. MacKillop J, Kahler CW. Delayed reward discounting predicts treatment response for heavy drinkers receiving smoking cessation treatment. Drug Alcohol Depend. 2009;104(3):197-203.
133. Krishnan-Sarin S, Reynolds B, Duhig AM, et al. Behavioral impulsivity predicts treatment outcome in a smoking cessation program for adolescent smokers. Drug Alcohol Depend. 2007;88(1):79-82.

134. Dallery J, Raiff BR. Delay discounting predicts cigarette smoking in a laboratory model of abstinence reinforcement. Psychopharmacology (Berl). 2007;190(4):485-96.

135. Muraven M. Practicing self-control lowers the risk of smoking lapse. Psychol Addict Behav. 2010;24(3):446-52.

136. Houben K, Wiers RW, Jansen A. Getting a grip on drinking behavior: training working memory to reduce alcohol abuse. Psychol Sci. 2011;22(7):968-75.

137. Houben K, Havermans RC, Nederkoorn C, Jansen A. Beer a nogo: learning to stop responding to alcohol cues reduces alcohol intake via reduced affective associations rather than increased response inhibition. Addiction. 2012;107(7):1280-7.

138. Houben K, Nederkoorn C, Wiers RW, Jansen A. Resisting temptation: decreasing alcohol-related affect and drinking behavior by training response inhibition. Drug Alcohol Depend. 2011;116(13):132-6.

139. Jones A, Field M. The effects of cue-specific inhibition training on alcohol consumption in heavy social drinkers. Exp Clin Psychopharmacol. 2013;21(1):8-16.

140. Bell, R.P., J.J. Foxe, L.A. Ross, and H. Garavan: Intact inhibitory control processes in abstinent drug abusers (I): A functional neuroimaging study in former cocaine addicts. Neuropharmacology. doi:10.1016/j.neuropharm.2013.02.018.

141. Morie, K.P., H. Garavan, R.P. Bell, et al.: Intact inhibitory control processes in abstinent drug abusers (II): A high-density electrical mapping study in former cocaine and heroin addicts. Neuropharmacology. doi:10.1016/j.neuropharm.2013.02.023. 